\title{
BMJ Open Trends in HIV prevalence and risk behaviours among men who have sex with men from 2013 to 2017 in Nanjing, China: a consecutive cross-sectional survey
}

Zhengping Zhu, ${ }^{1}$ Hongjing Yan, ${ }^{2}$ Sushu Wu, ${ }^{1}$ Yuanyuan $\mathrm{Xu},{ }^{1}$ Wenjiong $\mathrm{Xu},{ }^{1} \mathrm{Li} \mathrm{Liu},{ }^{1}$ Xin Li, ${ }^{1}$ Fei Xu, ${ }^{1}$ Roger Detels ${ }^{3}$

To cite: Zhu Z, Yan H, Wu S, et al. Trends in HIV prevalence and risk behaviours among men who have sex with men from 2013 to 2017 in Nanjing, China: a consecutive crosssectional survey. BMJ Open 2019;9:e021955. doi:10.1136/ bmjopen-2018-021955

- Prepublication history for this paper is available online. To view these files, please visit the journal online (http://dx.doi org/10.1136/bmjopen-2018021955).

Received 9 February 2018 Revised 22 August 2018 Accepted 8 October 2018

\section{Check for updates}

C Author(s) (or their employer(s)) 2019. Re-use permitted under CC BY-NC. No commercial re-use. See rights and permissions. Published by BMJ.

${ }^{1}$ Section of AIDS Control and Prevention, Nanjing Municipal Center for Disease Control and Prevention, Nanjing, China

${ }^{2}$ Section of AIDS Control and Prevention, Jiangsu Province Center for Disease Control and Prevention, Nanjing, China ${ }^{3}$ Department of Epidemiology, School of Public Health, University of California, Los Angeles, California, USA

Correspondence to Zhengping Zhu; zzp@njcdc.cn

\section{ABSTRACT}

Objective To examine the trends of HIV prevalence, risk behaviours and HIV testing among men who have sex with men (MSM) in Nanjing.

Design Five consecutive cross-sectional surveys.

Setting Nanjing, China.

Primary and secondary outcome measures HIV and syphilis prevalence, HIV testing rate and factors associated with HIV infection; demographic characteristics and behaviours.

Results 649, 669, 577, 633, 503 MSM were recruited from 2013 to 2017 . HIV prevalence was $9.9 \%, 12.3 \%$, $12.5 \%, 9.8 \%$ and $10.1 \%$, respectively. Syphilis prevalence decreased with a range from $10.6 \%$ to $5.6 \%$. Risk behaviours like unprotected anal intercourse (UAl) and unprotected virginal sex in the past 6 months decreased, but multiple sex partners and ever used rush popper rose significantly. MSM tested for HIV in the previous year remained stable from $57.0 \%$ to $64.1 \%$ ( $P=0.633$ ). Multivariate analysis showed that tested for HIV in the past year was protective factor against HIV infection. MSM who had UAl in the past 6 months, sex role as receptive and dual, diagnosed with sexually transmitted diseases (STDs) in the past year and currently syphilis infected were risk factors for HIV infection.

Conclusions We observed stable high HIV prevalence, a steady HIV testing rate, decreasing syphilis prevalence and UAI among MSM in Nanjing. However, rush popper use rose dramatically. The HIV preventive strategies for MSM including condom promotion, HIV testing expansion and reduction of rush popper use, STDs screening and standardised treatment should be strengthened.

\section{INTRODUCTION}

In recent years, a fast-spreading HIV epidemic among men who have sex with men (MSM) presented a new challenge worldwide. ${ }^{1}{ }^{2}$ In China, MSM transmission has surpassed both injection drug use and blood donors and has become the major HIV transmission route. ${ }^{34}$ The overall HIV prevalence, from national MSM sentinel surveillance data,

\section{Strengths and limitations of this study}

- The present study is designed to assess the recent HIV prevalence, risk behaviours and HIV testing trends among men who have sex with men (MSM) in Nanjing.

- We analysed and confirmed several factors associated with HIV infection using pooled 5 years' data.

- Rush popper use among Nanjing MSM had a rising trend.

- Snow ball sampling and internet convenience sampling may not yield a representative sample of the general MSM population; these data should be interpreted with caution.

- Face-to-face investigation may under evaluate risk behaviours due to the issue of social desirability.

demonstrated a rising trend from $0.9 \%$ in 2003 to $6.3 \%$ in $2011 .{ }^{5}$

Nanjing is the capital city of Jiangsu Province, eastern China, with a total population of 8.6 million. Consistent with many other metropolitan cities of China, homosexual transmission has become the major route of HIV transmission in Nanjing. ${ }^{6}$ According to the national direct network HIV/AIDS Case Reporting System, the annual proportion of newly reported HIV cases attributed to homosexual transmission rose from $44.3 \%$ in 2008 to $63.5 \%$ in $2012^{7}$ and to $71.3 \%$ in 2015 in Nanjing. Recent studies have highlighted a higher prevalence of club drugs use among $\mathrm{MSM}^{8}$, and rush popper (inhalant nitrites) is the most popular. Research has documented that rush popper use may increase HIV transmission by increasing engagement in high risk sex behaviours among MSM. ${ }^{9}$

In response to the escalating HIV epidemic among MSM, Nanjing implemented comprehensive prevention strategies over the past 
decade. Condom promotion and lubricant distribution were free in MSM popular venues. Almost 40 voluntary HIV testing and counselling sites were established across the city to provide friendly HIV testing service. From 2008 to 2012, under the implementing of China-Bill Melinda Gates Foundation on HIV/AIDS programme and the Global Fund to Fight AIDS programme in Nanjing, a growing number of MSM Community Based Organisations (CBOs) established and provided peer-led HIV rapid testing and referrals to treatment.

In 2013, the Chinese national centre for AIDS/sexually transmitted diseases (STDs) control and prevention launched Expanding HIV Testing and Scale-up Antiretroviral Therapy (ART) Programme in eight cities over the country, Nanjing was one of them. The programme implemented various measures. On one hand, Centers for Disease Control and Prevention (CDC) led health education programmes, HIV testing days, referrals for ART and standardised STDs treatment. On the other hand, CBOs carried out condom and lubricant distribution, behavioural interventions and provision of HIV rapid testing kits. After each test, staff of $\mathrm{CDC}$ or $\mathrm{CBO}$ provided risk-reduction counselling.

However, it is unknown whether these strategies reduced the HIV prevalence among MSM, and to what extent their risk behaviours were changed. Therefore, we designed and conducted five consecutive cross-sectional surveys from 2013 to 2017, aimed to identify the trends of HIV and syphilis prevalence, risk behaviours and HIV testing among MSM. We also analysed the factors correlated with HIV infection using the pooled 5years' survey data.

\section{METHODS}

\section{Participants}

Participants met the following criteria: 16 years of age or older, male, had oral or anal sex with a male in the previous year, could provide written informed consent and were willing to complete the study.

\section{Sampling method and participant recruitment}

We employed two methods to recruit participants: snow ball sampling and internet convenience sampling. For snow ball sampling, the participants were recruited from MSM venues such as bars, clubs, saunas, and public restrooms. Initial 'seed' participants were recommended by volunteers of CBOs or staffs of MSM bars. Each initial seed was invited to participate in the study and then asked to invite other person with the same inclusion criteria. We also posted study advertisements on some MSM social platforms and the Nanjing CDC official website, inviting MSM to participate in our study. These recruit processes continued from April to July in each year.

\section{Study procedures}

We designated two survey sites in Nanjing. They were VCT clinics in the Nanjing municipal CDC and Qinhuai district CDC. All interviewers were CDC staff members who were well trained. We screened for duplicate reported telephone numbers and excluded those repeated participants during a same survey year. The survey sites, main interviewers and recruit methods were consistent during the 5 years surveys.

After the qualification screening, every participant signed a consent form before the formal investigation. Face-to-face interviews were used to collect information including demographic characteristics, sexual behaviours in the past 6 months (with males and females), drugs use, STD diagnoses and HIV testing history. No name or identifying information was collected; $5 \mathrm{~mL}$ of whole blood was collected for HIV and syphilis test. After testing, we offered HIV risk-reduction counselling for each participant. Confirmed HIV cases were subsequently referred to the designated free ART clinic for treatment, and current syphilis cases were referred to standardised STDs clinics for treatment.

\section{Laboratory testing}

Blood samples were screened for HIV-1 antibody with a rapid test (Determine HIV-1/2, Alere Medical, Chiba Prefecture, Japan). Positive samples were retested by an enzyme-linked immunoassay (HIV Ag/Ab ELISA KIT 96T, Zhuhai Livzon Diagnostics, China), ELISA-positive cases were confirmed with a western blot assay (HIV BLOT 2.2, MP, Singapore). Syphilis screening was performed by Treponema pallidum particle assay (TPPA) (Alere Medical), and confirmed by the rapid plasma regain test (RPR) (Diagnosis; Shanghai, Kehua, China). TPPA-positive and RPR-positive participants were determined to be currently syphilis infected.

\section{Variable definitions}

'Unprotected anal intercourse (UAI)' was defined as inconsistent use of condoms during anal sex with male partners in the past 6 months; 'unprotected vaginal intercourse (UVI)' was defined as inconsistent use of condoms during vaginal sex with female partners in the past 6 months. We defined 'multiple sexual partners' as having had two or more sexual partners in the past 6 months. 'Ever used drugs' were defined as ever used heroin or opium.

\section{Statistical analysis}

Data were double entered and checked for accuracy using Epi Data software (V.3.0: Epi Data Association, Odense, Denmark). Descriptive statistics were used to describe participant's demographic characteristics and prevalence rate. Trend tests were performed using $\chi^{2}$ tests with linear-by-linear analysis. Univariate and multivariate logistic regression analyses were conducted to adjust ORs for potential confounding. Only variables that were significant in univariate analyses at $P<0.1$ were included in the multivariate logistic regression models. A $P$ value $<0.05$ (two-tailed) was considered to be statistically significant. 
Table 1 Demographic characteristics among men who have sex with men in Nanjing, 2013-2017

\begin{tabular}{|c|c|c|c|c|c|c|c|}
\hline Variables & 2013 & 2014 & 2015 & 2016 & 2017 & $\begin{array}{l}\text { Linear-by- } \\
\text { linear } \chi^{2}\end{array}$ & $P$ value \\
\hline Total sample & 649 & 669 & 577 & 633 & 503 & - & - \\
\hline$<20$ & $6.0(39)$ & $4.3(29)$ & $5.4(31)$ & $2.0(18)$ & $3.8(20)$ & & \\
\hline $20 \sim 49$ & $86.0(558)$ & $84.9(568)$ & $89.1(514)$ & $91.0(557)$ & $89.1(467)$ & & \\
\hline Single, divorced or widowed & $73.3(476)$ & 74.6 (499) & $81.3(469)$ & $81.5(516)$ & $84.5(425)$ & & \\
\hline Married or live together & $26.7(173)$ & $25.4(170)$ & $18.7(108)$ & $18.5(117)$ & $15.5(78)$ & & \\
\hline Han ethnic & $97.4(632)$ & $98.7(660)$ & $97.9(565)$ & $98.6(624)$ & $97.0(488)$ & 0.074 & 0.785 \\
\hline Residency (hukou) in Jiangsu & $68.4(444)$ & $71.4(478)$ & $66.7(385)$ & $69.0(437)$ & $67.6(340)$ & 0.437 & 0.509 \\
\hline College and higher & $57.6(374)$ & $64.7(433)$ & $67.8(391)$ & $69.7(441)$ & $68.6(345)$ & & \\
\hline Sex orientation & & & & & & 1.303 & 0.2524 \\
\hline Homosexual/bisexual & $94.1(611)$ & $93.6(626)$ & $92.7(535)$ & $94.8(600)$ & $95.4(480)$ & & \\
\hline Heterosexual/not sure & $5.9(38)$ & $6.4(43)$ & $7.3(42)$ & $5.2(33)$ & $4.6(23)$ & & \\
\hline
\end{tabular}

All statistical analyses were conducted using SPSS software (V.20; IBM, Armonk, New York, USA).

\section{Patient involvement}

Patients were not involved in setting the research question, the outcome measures, the design or the implementation of the study. No patients were asked to advise on interpretation or writing up of results. No patients were advised on dissemination of the present study and its main results.

\section{RESULTS}

\section{Demographic and social characteristics}

Demographic characteristics of the participants in the 5 -year surveys are presented in table 1 . The number of self-reported MSM was 649, 669, 577, 633 and 503, respectively. The composition of the samples and their corresponding characteristics were comparable in the five surveys. The majority of participants were aged 20 49 years, single, Han ethnicity, Jiangsu residents and highly educated. Over $90 \%$ of participants were either homosexual or bisexual. Significantly more MSM were single and highly educated.

\section{Trends in HIV prevalence, risk behaviours and HIV testing}

The trends of HIV prevalence, sexual behaviours, STD diagnosis and HIV testing rates among MSM are presented in table 2. The HIV prevalence among MSM range from $9.9 \%$ to $12.5 \%$, the trend was not significant. There was a decreasing trend of current syphilis prevalence with the range from $10.6 \%$ to $5.6 \%$. During the 5 years of the study, some risk behaviours like UAI, vaginal sex and
UVI in the past 6 months showed decreasing trends. On the other hand, the rate of multiple sexual partners and ever used rush popper indicated significantly increasing trends. The behaviours including anal sex, commercial anal sex, ever used drugs, diagnosed with STDs and tested for HIV showed no significant change.

\section{Factors associated with HIV infection}

A total of 3031 participants were included. Table 3 presents the results of unvariable logistic analyses of factors related to HIV infection among participating MSM. Factors with $p$ values $<0.10$ in univariable analysis were included in the multivariable model. Because the factors of 'anal sex' and 'UAI' in the past 6 months were correlated with each other $(\mathrm{r}=0.400, p<0.001)$, 'anal sex' was not included in multivariate logistic analysis. 'Ever used rush popper' was also excluded from multivariate logistic analysis for missing data in 2013. Table 4 shows the results of multivariate logistic regression analysis. Participants were more likely to be infected with HIV if they had UAI in the past 6 months, sex role as receptive or dual, diagnosed with STDs in the previous year and currently were syphilis infected. Participants tested for HIV in the previous year were less likely to be HIV infected compared with those not tested.

\section{DISCUSSION}

Over the five annual cross-sectional surveys, we found that HIV prevalence among MSM in Nanjing remained steady at a high level from 2013 to 2017. This trend is different from the period of 2008 to 2012 when a rapid increase 
Table 2 Trends in HIV prevalence, risk behaviours and HIV testing among MSM in Nanjing, 2013-2017

\begin{tabular}{|c|c|c|c|c|c|c|c|}
\hline \multirow[b]{2}{*}{ Variables } & \multirow{2}{*}{$\begin{array}{l}2013 \\
\%(n) \\
\end{array}$} & \multirow{2}{*}{$\begin{array}{l}2014 \\
\%(n) \\
\end{array}$} & \multirow{2}{*}{$\begin{array}{l}2015 \\
\%(n) \\
\end{array}$} & \multirow{2}{*}{$\begin{array}{l}2016 \\
\%(n) \\
\end{array}$} & \multirow{2}{*}{$\begin{array}{l}2017 \\
\% \text { (n) }\end{array}$} & \multirow{2}{*}{$\begin{array}{l}\text { Linear- } \\
\text { by-linear } \\
\chi^{2}\end{array}$} & \multirow{2}{*}{$P$ value } \\
\hline & & & & & & & \\
\hline HIV infection & $9.9(64)$ & $12.3(82)$ & $12.5(72)$ & $9.8(62)$ & $10.1(51)$ & 0.181 & 0.670 \\
\hline Current syphilis infection & $6.2(40)$ & $10.6(71)$ & $5.9(34)$ & $4.7(30)$ & $5.6(28)$ & 5.043 & 0.025 \\
\hline Had anal sex, last 6 months & 86.1 (559) & $81.0(542)$ & $76.4(441)$ & $83.6(529)$ & $80.7(406)$ & 2.958 & 0.085 \\
\hline Had UAl, last 6 months & 50.7 (329) & $42.5(284)$ & $43.0(248)$ & $41.2(261)$ & $41.7(210)$ & 9.433 & 0.002 \\
\hline Multiple sex partners, last 6 months & $55.6(361)$ & $49.6(332)$ & $46.3(267)$ & $57.3(363)$ & $62.0(312)$ & 19.194 & $<0.001$ \\
\hline Had commercial anal sex, last 6 months & $2.8(18)$ & $3.6(24)$ & $3.1(18)$ & $5.6(17)$ & $4.4(18)$ & 3.397 & 0.065 \\
\hline Had vaginal sex, last 6 months & $22.2(144)$ & $22.4(150)$ & $19.2(111)$ & $19.9(126)$ & $16.3(82)$ & 7.034 & 0.008 \\
\hline Had UVI, last 6 months & $14.0(91)$ & $15.1(101)$ & $11.6(67)$ & $13.7(87)$ & $9.1(46)$ & 6.791 & 0.009 \\
\hline Ever used rush popper* & - & $12.9(86)$ & $20.8(120)$ & $23.1(146)$ & $21.7(109)$ & 17.879 & $<0.001$ \\
\hline Ever used drugs & $2.0(13)$ & $0.9(6)$ & $1.7(10)$ & $0.9(6)$ & $1.2(6)$ & 1.166 & 0.280 \\
\hline $\begin{array}{l}\text { Diagnosed with sexually transmitted } \\
\text { disease, last } 12 \text { months }\end{array}$ & $6.9(45)$ & $6.6(44)$ & $4.7(27)$ & $8.4(53)$ & $7.0(35)$ & 0.333 & 0.564 \\
\hline Tested for HIV, last 12 months & $61.2(397)$ & $64.1(429)$ & $57.0(329)$ & $63.2(400)$ & $63.4(319)$ & 0.228 & 0.633 \\
\hline
\end{tabular}

*The data of 'ever used rush poppers' have not been collected in 2013.

MSM, men who have sex with men; UAI, unprotected anal intercourse; UVI, unprotected vaginal intercourse.

from $6.6 \%$ (RDS sampling, 430 sample size) ${ }^{10}$ to $13.7 \%$ (snow ball sampling, 670 sample size) ${ }^{11}$ were reported. These surveys were conducted by the same work team and recruited from the same venues. We also observed almost $10 \%$ decline in UAI among MSM. These outcomes should be cautiously attributed to the comprehensive prevention programme implementation, especially the supply of risk-reduction counselling after each test. Compared with other cities, HIV prevalence among MSM in Nanjing was much lower than in the southwestern cities of Chongqing $(21.2 \% \text { in 2014) })^{12}$ and Kunming (17.0\% in 2014). ${ }^{13}$ It was higher than in Beijing ${ }^{14}(6.1 \%$ in 2011$)$ or other countries (eg, $4.4 \%$ in India ${ }^{15}$ in $2010,2.5 \%$ in the Republic of Cyprus ${ }^{16}$ in 2012). However, all of these comparisons should be considered cautiously as there were differences in methodologies used to estimate prevalence. Because HIV continues to spread in Nanjing, interventions should be continued and strengthened.

We found that the HIV testing rate in the previous year remained steady at around $60 \%$, higher than that reported in Chongqing (43.5\% in 2014), ${ }^{12}$ Beijing $\left(42.6 \%\right.$ in 2016) ${ }^{17}$ and Guangzhou (53.4\% in 2013). ${ }^{18}$ It was also higher than the national annual HIV testing rate (50.4\% in 2011) among MSM. ${ }^{19}$ HIV testing rate among MSM in Nanjing increased from only one in five in 2008 to nearly half in $2012,{ }^{20}$ and then to almost two-thirds by 2017. The disclosure of HIV-positive status can have individual health benefits and potentially reduce community spread of HIV through early diagnosis and early initiation of ART. ${ }^{21}$ Our multivariate analysis confirmed that being tested for HIV in the previous year was a protective factor. However, there is still a gap in HIV testing rate among MSM between Nanjing and other areas, like Australia
$(80.5 \%)^{22}$ and the USA $(67 \%) .{ }^{23}$ We need to continue the expanding test programme further.

It is alarming that rush popper use among MSM in Nanjing rose dramatically from $12.4 \%$ to $21.7 \%$, it is just a little bit lower than that reported by Beijing $(26.8 \%)^{24}$ or Shenyang (26.3\%). ${ }^{25}$ Rush popper used to be a prescription drug prescribed to relieve angina. Now MSM is used to facilitate sexual intercourse due to its mechanism of relaxing the anal sphincter and dilating capillaries. ${ }^{26}$ Univariate analysis indicated that rush popper use was associated with a higher rate of HIV infection, which was consistent with other reports. ${ }^{24} 2527$ Two factors may explain this result. First, rush popper has the effects on sexual behaviours such as promoting sexual desire, reducing sexual inhibition and decreasing physical experiences of pain, which may further prevent users from using condoms. Second, prior research had documented that rush popper use may increase HIV transmission through their engagement in group sex and multiple sex partners. ${ }^{28}$ Our findings underscore the need for specific intervention programmes to reduce rush popper use.

We analysed the factors associated with HIV infection using the pooled five surveys data. Consistent with other reports, ${ }^{129}$ our data confirmed that UAI is an important risk factor for HIV infection. Although there was a declining trend of UAI among MSM, slightly $>40 \%$ of participants reported using condoms only intermittently or never. It is necessary to improve awareness of the risk of HIV infection among MSM and the need for consistent condom use during sexual activity. In the present study, anal sex role was found to be independent risk factor for HIV infection men who engaged exclusively or partially in receptive anal sex carry a higher risk of HIV infection, 
Table 3 Univariate analysis of factors associated with HIV infection among MSM in Nanjing

Factors HIV infection \% (n)

OR $(95 \% \mathrm{Cl})$

$P$ value

Age group (years)

\begin{tabular}{|c|c|c|c|}
\hline$<20$ & $6.6(9)$ & 1.000 & \\
\hline $20 \sim 49$ & $11.1(297)$ & 1.785 (0.898 to 3.546$)$ & 0.098 \\
\hline$\geqq 50$ & $10.9(25)$ & $1.734(0.785$ to 3.834$)$ & 0.174 \\
\hline \multicolumn{4}{|c|}{ Residency (hukou) } \\
\hline Jiangsu & $11.1(231)$ & 1.000 & \\
\hline Others & $10.6(100)$ & $0.947(0.739$ to 1.214$)$ & 0.668 \\
\hline
\end{tabular}

Education level

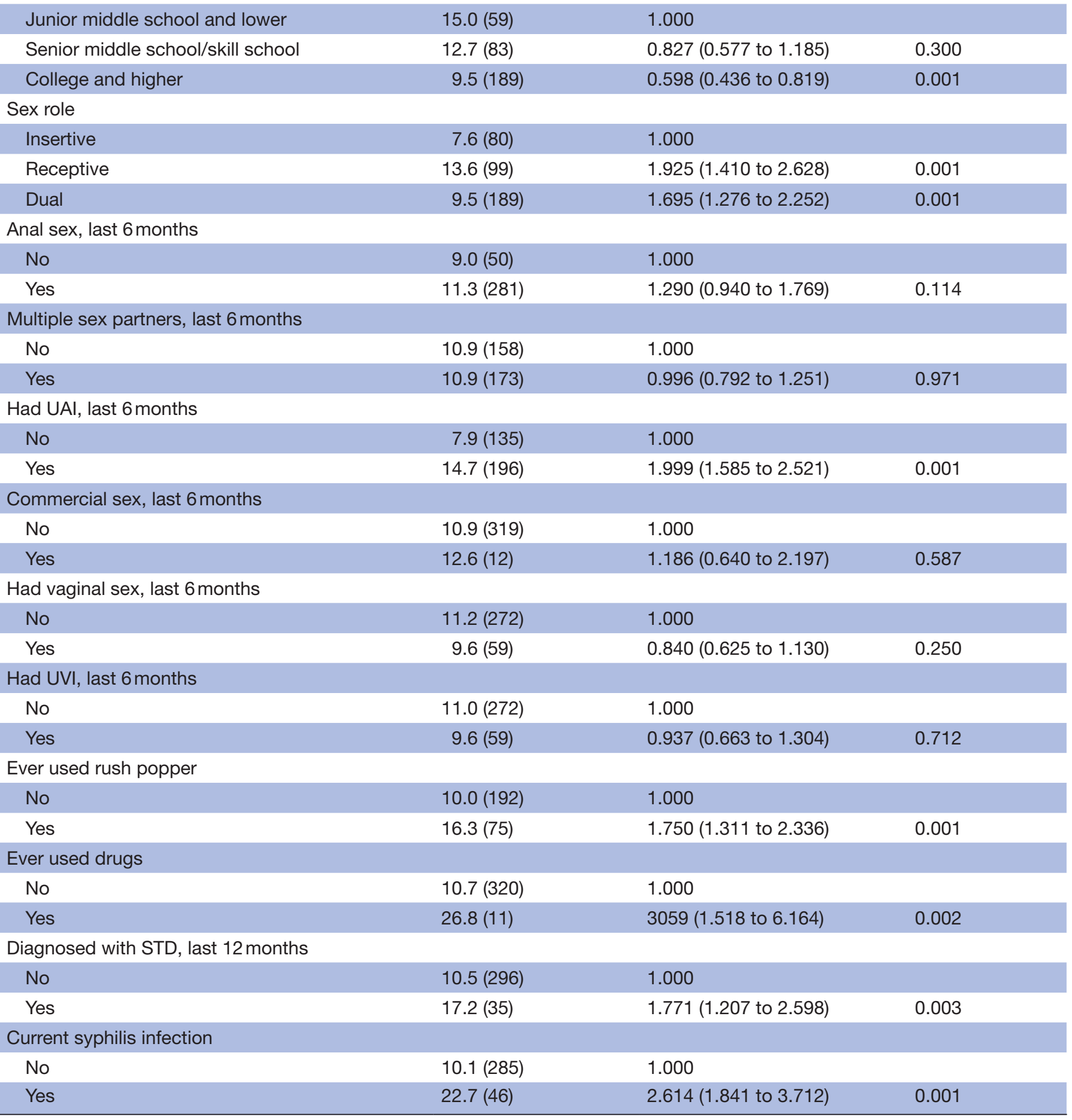


Table 3 Continued

\begin{tabular}{lccc}
\hline Factors & HIV infection \% (n) & OR (95\% Cl) & $P$ value \\
\hline Tested for HIV, last 12 months & & & \\
No & $12.9(149)$ & 1.000 & $0.728(0.578$ to 0.916$)$ \\
Yes & $9.7(182)$ & 0.007 & \\
\hline
\end{tabular}

MSM, men who have sex with men; STD, sexually transmitted disease; UAI, unprotected anal intercourse; UVI, unprotected vaginal intercourse.

possibly because rectal mucosa is easily damaged during receptive anal sex, thus increasing the likelihood of HIV virus passing into their blood. ${ }^{30}$ Therefore, both the 'receptive' and 'dual' role MSM should probably be considered a priority target for condom promotion.

We observed a declining trend in current syphilis prevalence among MSM during these recent 5 years. Compared

Table 4 Multivarible analysis of factors associated with HIV infection among MSM in Nanjing

\begin{tabular}{|c|c|c|}
\hline Factors & OR $(95 \% \mathrm{Cl})$ & $P$ value \\
\hline \multicolumn{3}{|l|}{ Education level } \\
\hline $\begin{array}{l}\text { Junior middle school } \\
\text { and lower }\end{array}$ & 1.000 & \\
\hline $\begin{array}{l}\text { Senior middle school/ } \\
\text { skill school }\end{array}$ & $0.883(0.610$ to 1.278$)$ & 0.509 \\
\hline College and higher & 0.700 (0.503 to 1.035$)$ & 0.501 \\
\hline \multicolumn{3}{|l|}{ Sex role } \\
\hline Insertive & 1.000 & \\
\hline Receptive & 1.936 (1.409 to 2.660$)$ & 0.001 \\
\hline Dual & 1.684 (1.261 to 2.249$)$ & 0.001 \\
\hline \multicolumn{3}{|l|}{ Had UAI, last 6 months } \\
\hline No & 1.000 & \\
\hline Yes & 2.046 (1.558 to 2.687$)$ & 0.001 \\
\hline \multicolumn{3}{|l|}{ Ever used drugs } \\
\hline No & 1.000 & \\
\hline Yes & $1.874(0.997$ to 4.120$)$ & 0.053 \\
\hline \multicolumn{3}{|c|}{ Diagnosed with STD, last 12 months } \\
\hline No & 1.000 & \\
\hline Yes & 1.610 (1.077 to 2.407$)$ & 0.020 \\
\hline \multicolumn{3}{|l|}{ Current syphilis infection } \\
\hline No & 1.000 & \\
\hline Yes & $2.219(1.531$ to 3.217$)$ & 0.001 \\
\hline \multicolumn{3}{|c|}{ Tested for HIV, last 12 months } \\
\hline No & 1.000 & \\
\hline Yes & 0.631 (0.437 to 0.912$)$ & 0.014 \\
\hline
\end{tabular}

The variables that included in multivariable analysis were below: education level, sex role, had UAI, ever used drugs, diagnosed with STD in last 12 months, current syphilis infection and test for HIV in last 12 months.

MSM, men who have sex with men; STD, sexually transmitted disease; UAI, unprotected anal intercourse. with previous report, ${ }^{11}$ current syphilis prevalence was lower than that in $2011(9.1 \%)$ and $2012(11.5 \%)$. This phenomenon is consistent with the report that syphilis infection has declined in China. ${ }^{30}$ The implementation of the syphilis prevention and control plan by the China's Ministry of Health may have contributed to this decline. ${ }^{31}$ It is well known that STDs that cause ulcers or inflammation greatly increase the efficiency of HIV transmission, by increasing both the infectiousness and the susceptibility to HIV infection. ${ }^{32}$ The positive association observed between syphilis and HIV can be explained by similar risk behaviours. ${ }^{33}$ Thus, anyone presenting with syphilis should be tested for HIV and vice versa. ${ }^{34}$ Meanwhile, screening and standardising STDs treatment need to be continued to push this trend further.

Several limitations of our study should be noted. First, our participants were recruited from MSM venues, some MSM social platforms and a government internet site. Thus, they may not be representative of MSM who do not go to these venues or visit the website. 'Hidden' MSM may carry higher risk behaviours. Second, it is possible that face-to-face investigation may have underestimated the levels of risk behaviours due to the issue of social desirability. The study was however anonymous; fake names and study serial number were used to match the records. Third, our cross-sectional studies are inherently observational and descriptive; thus, we cannot infer causality.

Overall, our five consecutive surveys were carefully designed, implemented and quality controlled. Under the implementation of comprehensive interventions, we observed stable HIV prevalence, steady HIV testing rate, decreasing UAI and syphilis prevalence. However, an increasing use of rush popper was observed. In response to the high HIV burden among MSM in Nanjing, HIV prevention and intervention messages must be increased about the urgent need for consistent condom use, targeting especially those MSM who engage in any receptive anal intercourse (although condom use is also important for inserters), HIV testing expanded, rush popper use reduced, STD screening increased and more widespread use of standardised treatment implemented.

Acknowledgements The authors are grateful to all the dedicated fieldworkers who took part in the surveys and all MSM participants who accepted the questionnaire investigation and blood sample providing. The authors would also like to thank MSM CBOs including: Nanjing Xingyou Community Based Organization, Nanjing Yeshanteng Bathroom Community Based Organization, Nanjing Bibochi Bathroom Community Based Organization and Nanjing Compass work studio. 
Contributors SW, YX, LL, XL were contributed to data collection. WX contributed to laboratory testing, FX contributed to the study design and quality control, ZZ was responsible for data analysis and manuscript writing, $\mathrm{HY}$ and $\mathrm{RD}$ contributed to manuscript revision. All authors read and approved the final manuscript.

Funding The study was supported by outstanding Medical Academic Leader Programme of Jiangsu Provincial Center for Disease Control and Prevention (grant no. JKRC2011003), Jiangsu Department of Health Programme (grant no. H201330) and Nanjing Field Epidemiology Training Programme (first session).

Competing interests None declared.

Patient consent Not required.

Ethics approval This study was approved by the Ethics Committee of the National Center for AIDS and STD Control, China CDC.

Provenance and peer review Not commissioned; externally peer reviewed.

Data sharing statement № additional data are available.

Open access This is an open access article distributed in accordance with the Creative Commons Attribution Non Commercial (CC BY-NC 4.0) license, which permits others to distribute, remix, adapt, build upon this work non-commercially, and license their derivative works on different terms, provided the original work is properly cited, appropriate credit is given, any changes made indicated, and the use is non-commercial. See: http://creativecommons.org/licenses/by-nc/4.0/.

\section{REFERENCES}

1. Beyrer C, Baral SD, van Griensven F, et al. Global epidemiology of HIV infection in men who have sex with men. Lancet 2012;380:367-77.

2. UNAIDS. UNAIDS World AIDS DAY Report, 2012.

3. China Ministry of Health. The Chinese Health Minister: men who have sex with men have become high risk population of HIV infection in China. Beijing: China Ministry of Health, 2009.

4. Ministry of Health, People's Republic of China, Joint United Nations Program on HIV/AIDS, World Health Organization. 2011 estimates for the HIV/AIDS epidemic in China, Beijing, China. 2011.

5. Wang L, Wang L, Norris JL, et al. HIV prevalence and influencing factors analysis of sentinel surveillance among men who have sex with men in China, 2003 - 2011. Chin Med J 2012;125:1857-61.

6. Zhu ZP, Zhang M, Ss W, et al. Analysis on epidemiological characteristics of reported HIV/AIDS infected people in Nanjing city, 2008-2012. Chin J Dis Control Prev 2013;17:1037-40.

7. Guan W, Zhu Y, Wei Q, et al. [Trends on the changing prevalence in patients with early syphilis and HIV infection among men who having sex with men in Nanjing, from 2008 to 2013]. Chin j Epidemiol 2015;36:624-8.

8. Reback CJ, Fletcher JB, Shoptaw S, et al. Methamphetamine and other substance use trends among street-recruited men who have sex with men, from 2008 to 2011. Drug Alcohol Depend 2013;133:262-5.

9. Phillips G, Magnus M, Kuo I, et al. Correlates of group sex among a community-based sample of men who have sex with men (MSM) in Washington, DC. AIDS Behav 2014;18:1413-9.

10. Yang $\mathrm{H}$, Hao $\mathrm{C}$, Huan $\mathrm{X}$, et al. HIV incidence and associated factors in a cohort of men who have sex with men in Nanjing, China. Sex Transm Dis 2010;37:208-18.

11. Yy X, Zhu ZP, Ss W, et al. Infection status of HIV in men who have sex with men in Nanjing, 2011-2015. Chin j Epidemiol 2016;37:1503-8.

12. Zeng $X$, Zhong $X$, Peng $B$, et al. Prevalence and associated risk characteristics of HIV infection based on anal sexual role among men who have sex with men: a multi-city cross-sectional study in Western China. Int J Infect Dis 2016;49:111-8.

13. Zhang $X$, Jia $M$, Chen $M$, et al. Prevalence and the associated risk factors of HIV, STIs and HBV among men who have sex with men in Kunming, China. Int J STD AIDS 2017;28:1115-23.
14. Han Y, Xia DY, Sun YM, et al. HIV prevalence and its related factors among men who have sex with men in Beijing. Chin J AIDS STD 2013;19:399-401.

15. Jha UM, Raj Y, Venkatesh S, et al. HIV epidemic among men who have sex with men in India: national scenario of an unfinished agenda. Hiv Aids 2014;6:159-70.

16. Pylli M, Middleton N, Charalambous A, et al. HIV prevalence, sexual and HIV testing behaviors among men who have sex with men in the Republic of Cyprus: 2011-2012 data from a cross-sectional study. BMC Infect Dis 2014;14:432.

17. DI L, Sun JY, Zhang Y, et al. HIV prevalence and related factors among men who have sex with men in Beijing. Chin Prev Me 2016;17:321-6.

18. Zhong $\mathrm{F}$, Liang $\mathrm{B}, \mathrm{Xu} \mathrm{H}$, et al. Increasing HIV and decreasing syphilis prevalence in a context of persistently high unprotected anal intercourse, six consecutive annual surveys among men who have sex with men in Guangzhou, China, 2008 to 2013. PLoS One 2014;9:e103136.

19. State Council AIDS Working Committee Office (SCAWCO). 2012 China AIDS response progress report. Beijing, China: Ministry of Health of the People's Republic of China, 2012:1-70.

20. Yan H, Li J, Raymond HF, et al. Increased HIV testing among men who have sex with men from 2008 to 2012, Nanjing, China. PLoS One 2016;11:e0154466.

21. Zhao $\mathrm{Y}$, Zhang $\mathrm{L}$, Zhang $\mathrm{H}$, et al. HIV testing and preventive services accessibility among men who have sex with men at high risk of HIV infection in Beijing, China. Medicine 2015;94:e534.

22. Lyons A, Pitts M, Grierson J, et al. Sexual behavior and HIV testing among bisexual men: a nationwide comparison of Australian bisexual-identifying and gay-identifying men. AIDS Behav 2012;16:1934-43.

23. Centers for Disease Control and Prevention (CDC). HIV testing and risk behaviors among gay, bisexual, and other men who have sex with men - United States. MMWR Morb Mortal Wkly Rep 2013;62:958-62.

24. Zhang $\mathrm{H}$, Teng $\mathrm{T}$, Lu $\mathrm{H}$, et al. Poppers use and risky sexual behaviors among men who have sex with men in Beijing, China. Drug Alcohol Depend 2016;160:42-8.

25. Xu JJ, Zhang C, Hu QH, et al. Recreational drug use and risks of HIV and sexually transmitted infections among Chinese men who have sex with men: Mediation through multiple sexual partnerships. BMC Infect Dis 2014;14:642-9.

26. Romanelli F, Smith KM, Thornton AC, et al. Poppers: epidemiology and clinical management of inhaled nitrite abuse. Pharmacotherapy 2004;24:69-78.

27. Zhu ZP, Zhang M, Xu YY, et al. [Cross-sectional surveys on the use of recreational drug nitrous-acid-ester rush-poppers in men who have sex with men, Nanjing]. Zhonghua Liu Xing Bing Xue Za Zhi 2017;38:189-93.

28. Xu JJ, Qian HZ, Chu ZX, et al. Recreational drug use among Chinese men who have sex with men: a risky combination with unprotected sex for acquiring HIV infection. Biomed Res Int 2014;2014:1-9.

29. Pan $X$, Wu M, Ma Q, et al. High prevalence of HIV among men who have sex with men in Zhejiang, China: a respondent-driven sampling survey. BMJ Open 2015;5:e008466.

30. Chen Y, Tang W, Chen L, et al. Changing epidemic of HIV and syphilis among resident and migrant men who have sex with men in Jiangsu, China. Sci Rep 2017;7:9478.

31. Li X, Lu H, Cox C, et al. Changing the landscape of the HIV epidemic among MSM in China: results from three consecutive respondentdriven sampling surveys from 2009 to 2011. Biomed Res Int 2014;2014:1-10.

32. Galvin SR, Cohen MS. The role of sexually transmitted diseases in HIV transmission. Nat Rev Microbiol 2004;2:33-42.

33. Jin F, Jansson J, Law M, et al. Per-contact probability of HIV transmission in homosexual men in Sydney in the era of HAART. AIDS 2010;24:907-13.

34. Karumudi UR, Augenbraun M. Syphilis and HIV: a dangerous duo. Expert Rev Anti Infect Ther 2005;3:825-31. 